\title{
A New Distributed Approach for Range Image Segmentation
}

\author{
Smaine Mazouzi ${ }^{1}$ and Zahia Guessoum ${ }^{2}$ \\ 1 Dép. d'informatique, Université de Skikda, BP 26, Route ElHadaik, 21000, Algeria \\ smazouzi@univ-skikda.dz \\ 2 LIP6, Université de Paris 6, 104, av. du Président Kennedy, 75016, Paris, France \\ zahia.guessoum@lip6.fr
}

\begin{abstract}
In this paper we introduce a new distributed approach for image segmentation based on multi-agent systems. Several agents are placed randomly in the image, then each of them starts a region growing around its position. Several agents can be within the same homogeneous region. So, they must exchange information to better labeling pixels reached by these agents. Every labeled pixel is smoothed by replacing its parameters by those of the pixel in the center of the region seed. A set of real range images from the ABW image base was used to evaluate the proposed approach. Experimental results show the potential of the approach to provide an accurate and efficient image segmentation.
\end{abstract}

Keywords: Image segmentation, Multi-agent systems, Region growing.

\section{Introduction}

Segmenting an image is necessary to perform several tasks in image analysis and object recognition. Based on some similarity criterion, it consists in participating pixels of an image in homogeneous and contiguous sets, called regions. Mostly, image segmentation methods are divided in two categories: Edge-based methods, and region-based methods. In the first category, pixels corresponding to discontinuities in image information are selected [46]. After, the obtained pixels are chained and partitioned into disjoint sets to form boundaries of image regions. Edge-based methods are characterized by a low computational cost, suitable for real-time applications. However, they are sensitive to noise and distortions in images. Region-based methods use some homogeneity creterion in order to gather pixels in homogeneous and contiguous regions 31. Contrary to edge-based methods, region-based ones are time and memory costly, and their performances depend on the selection of the region seeds. Nevertheless, they provide better results even in presence of noise and distortions.

Most of authors having proposed multi-agent systems for image segmentation have opted for supervised approaches [2910117], where the number or the shape of the regions are beforehand fixed. So, these systems can be used only with images for that they were conceived. We propose through this work an unsupervised and distributed method, modeled as a multi-agent system, for image 
segmentation. We do not make any assumption on the number of the regions in considered images. The principle of the system consists in placing randomly in the image a high number of autonomous and situated agents. Each one of them proceeds for a region growing from a given seed, selected according to a quality criterion. So, many agents can coexist within the same region. In this case, they must exchange some informations in order to better label shared pixels. A given image region is successively smoothed by several agents which are situated within. This allow good surface fitting, that allow in its turn accurate region extraction. Moreover, in our case agents are weakly coupled, allowing the implementation of the proposed system on massively parallel computers.

The reminder of the paper is organized as follows: in Section 2 we start by introducing how surfaces are modeled in range images. The reminder of the Section 2 is devoted to the proposed approach. We introduce in this section the principle of the multi-agent system, and agent behavior, modeled as an Alive method within the agent. Experimental results and comparison are introduced through Section 3, in which we show the parameter selection, and the obtained results. Finally, a conclusion summarizes our paper, and underlines perspective work.

\section{Multi-agent Image Segmentation}

\subsection{Surface Modeling in Range Images}

In this work we have used range images for experimental purpose. However, the proposed approach can be used with any type of images, including 2-D and 3-D images. In a range image, each pixel $(x, y)$ memorizes the depth $d(x, y)$ spacing the range finder plane and the corresponding point of the scene. In order to define a homogeneity criterion allowing region growing, we use a new representation $\left(d^{*}\right)$ of the row image, where $d^{*}(x, y)$ represents the tangent plane to the surface at $(x, y)$. The tangent plane at $(x, y)$ is obtained by the multiple regression method using the set of neighboring pixels situated within a $3 \times 3$ window centred at $(x, y)$, and whose depths are close, according to a given threshold $\left(T r_{h}\right)$. The plane equation in a 3 -D coordinate system may be expressed as follows:

$$
z=a x+b y+c
$$

where $(a, b,-1)^{T}$ is a normal vector to the plane, and $|c| / \sqrt{a^{2}+b^{2}+1}$ is the orthogonal distance between the plane and the coordinate origin. We consider that a pixel belongs to a planar region, given its plane equation, if the distance $(h)$ between the respective planes is less than $\left(T r_{h}\right)$, and the angle $(\phi)$ between the respective normals is less than $T r_{\phi}$, where $T r_{h}$ and $T r_{\phi}$ are respectively the distance and the angle thresholds. The quality of plane estimation $q(x, y)$ at $(x, y)$ according to the regression model is also computed. The latter is used to accept or reject a region seed when an agent is initialized at a random position in the image. 


\subsection{Agent Behavior}

A population of 1500 situated agents are randomly placed in the image. Each agent performs a region growing starting from its position. Contrary to classical region growing, where pixels are labeled as certainly homogeneous pixels, each pixel in our case is labeled as homogeneous pixel with certainly degree. This latter is expressed by the angle between the normal vector at the pixel in question, and the normal vector at the center of the region seed. The agent writes also its identifier at the current pixel. So, each homogenous pixel memorizes the identifier of the last agent having included the pixel into its homogeneous region.

At the creation of an agent, this latter examines its neighborhood in order to decides if it can be a region seed, from where it starts a region growing. For this, the agent calculates the estimation quality $(q)$ according to the multiple regression model.

$$
q=\frac{\sum_{i \in \text { Seed }}\left(\widehat{z_{i}}-\bar{z}\right)^{2}}{\sum_{i \in \text { Seed }}\left(z_{i}-\bar{z}\right)^{2}}
$$

where $\widehat{z_{i}}$ and $z_{i}$ are respectively the estimated and the measured range value of the pixel $i$ in the seed.

If the estimation quality $q$ is greater than a given threshold $Q$, the agent performs a region growing from the given seed, by iteratively including the homogeneous pixels at the current borders of the region in growth. At each homogeneous reached pixels which is not yet labeled (by any other agent), the agent writes its identifier, the angle $\phi$ between the normal vector to the surface at the current pixel and the normal vector to the surface at the seed center. However, if the reached pixel has been labeled before, the agent, lets called $A$, initiates a communication with the agent, lets called $B$, whom has last labeled the pixel. The agent $A$ requests needed informations form the agent $B$ in order decide if the label of the pixel must be set, or left as it is (Fig. 1). The information needed consists in the size of the region corresponding to the agent $B$.

The agent $A$ makes decision according to several parameters which are : sizes of the two regions $\left(S_{i z e_{A}}, S i z e_{B}\right)$ and the angles between respectively the normal surface vectors at the pixel and the region seeds $\left(\phi_{A}, \phi_{B}\right)$. So, the label which it was $(B)$ is set to $(A)$ if :

$$
\phi_{A}+\eta \times \operatorname{Size}_{A}<\phi_{B}+\eta \times \operatorname{Size}_{B}
$$

with $\eta$ a constant parameter set at the parameter selection (see Section 3.1). In this case the angle $\phi_{B}$, memorized at the position of the pixel, is set to $\phi_{A}$.

At each reached homogeneous pixel, the image is smoothed by replacing the parameters $(a, b, c)$ of the plane at the pixel with those of the region seed center.

The method Alive, introduced below, represents the behavior of a given agent. The job on an agent can be interrupted by an information request. In this case the interrupted agent responds by sending the current region size via the IncomingRequest event. Then, it returns to continue with the Alive thread.

The agent environnement is formed by the Image array $\left(d^{*}\right)$ and a second array called Labels, both of ImageSize ${ }^{2}$ elements. Each element in Labels array 

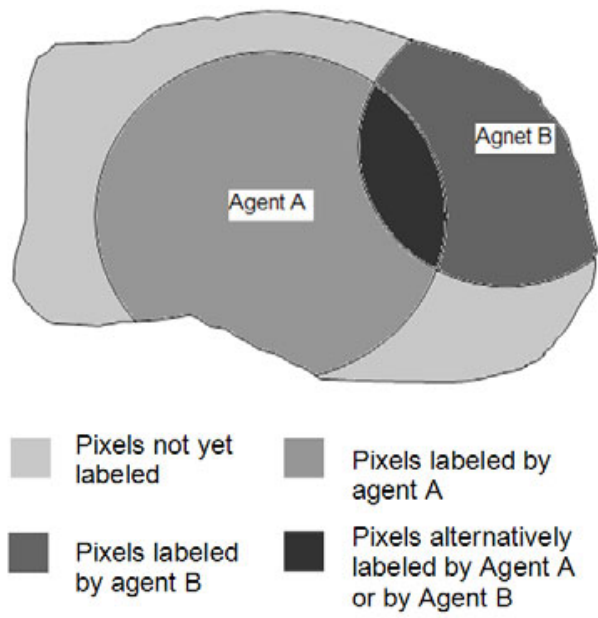

Fig. 1. Two agents growing the same region. Pixels are labeled A by Agent A, or B by Agent B according to region sizes and to angles between normal vectors.

memorizes an agent identifier (the last has labeled the pixel), and the angle between the normal vector at the pixel and the normal vector at the seed center. At any time an agent can receive a request for information, as an event. It then responds the sender by sending its current region size.

According to this behavior of agents, a given planar region is smoothed and labeled by several agents (belonging all to the same region). The way by which pixels are smoothed allows a smart image denoising, given the fact that pixels of the same homogeneous region are smoothed by considering the best region seed.

\section{Experimentation}

Most of authors having dealt with range images 6831 have used a dedicated framework for performance evaluation of range image segmentation algorithms, proposed by Hoover et al. [5].

According to this framework a machine-generated segmentation (MS) is compared to an ideal segmentation, which represents a ground truth (GT). An extracted region can be classified as a correct detection, an over-segmentation, an under-segmentation, a missed region, or a noise region. A compare tool tolerance $T ; 50 \%<T \leq 100 \%$ is used to express the strictness of the classification. The 40 real images of ABW set are divided into two subsets: 10 training images, and 30 test images. Four methods, namely USF, WSU, UB and UE, cited in [5] are involved in the result comparison.

\subsection{Parameter Selection}

The set of training images with their ground truth segmentation is used according to a supervised learning approach, in order to select optimal values of 

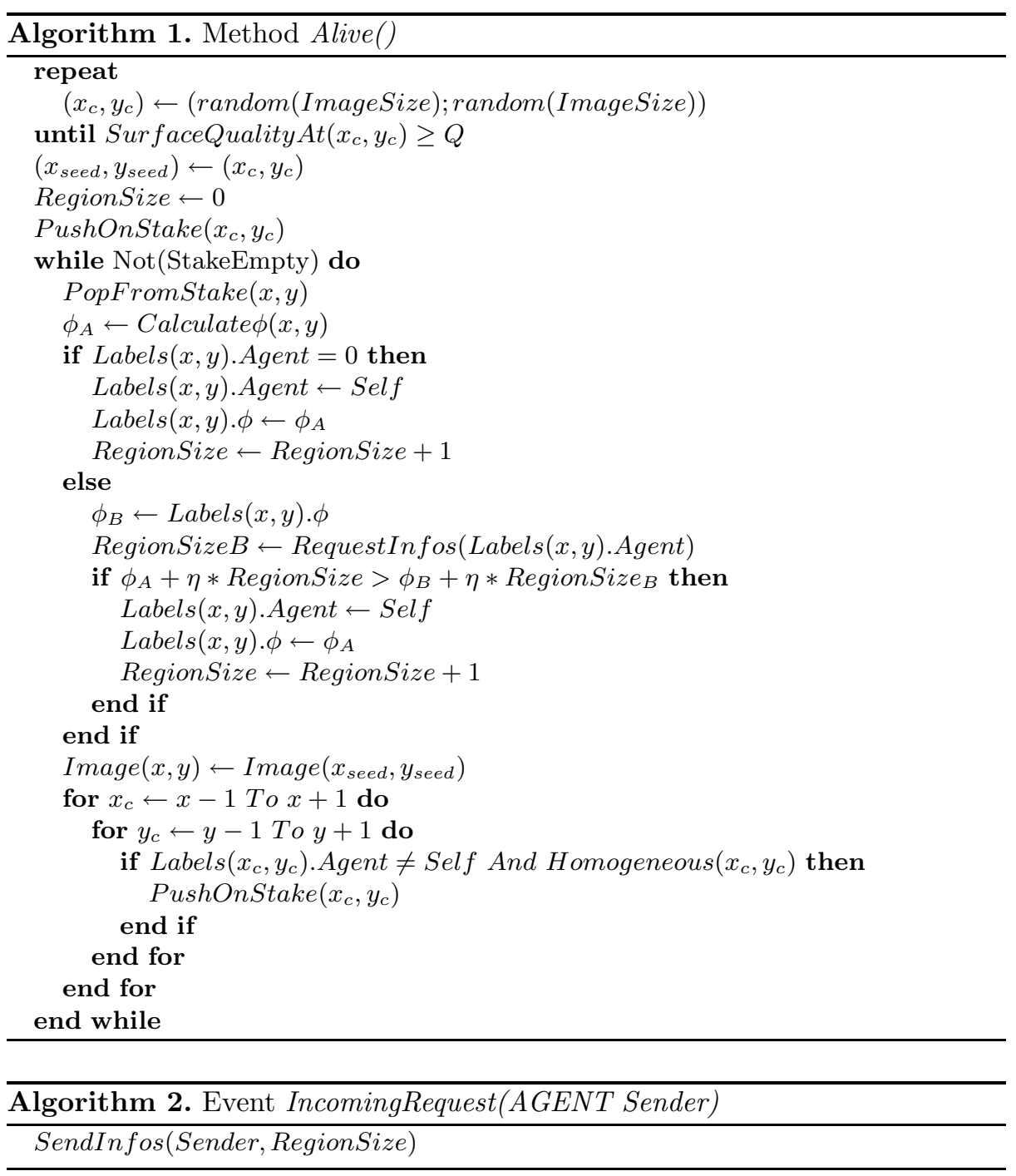

the involved parameters. Optimal parameter values correspond to the maximum of regions correctly detected, according to a ground truth (GT), with $T$ set to $80 \%$ [5].

Our method, named MABIS for Multi-Agent-Based Image Segmentation uses four parameters, for which optimal values must be set. Namely they are $T r_{\phi}$, $\operatorname{Tr}_{h}, Q$, and $\eta$ (see Section 2). The value of $\eta$ is simply set to $1 / R S A$, where $R S A$ is the average of sizes of all regions in the training set. For the reminder of parameters, 64 combinations namely $\left(\operatorname{Tr}_{\phi}, \operatorname{Tr}_{h}, Q\right) \in\left\{12^{\circ}, 15^{\circ}, 18^{\circ}, 21^{\circ}\right\} \times$ $\{12,16,20,24\} \times\{0.90,0.95,0.97,0.99\}$, were run on the training set. Obtained optimal values of the parameters, which correspond to the maximum correct 
detection for the overall training set of range images, are as follows: $\operatorname{Tr}_{\phi}=18^{\circ}$, $\operatorname{Tr}_{h}=16$, and $Q=0.95$.

\subsection{Experimental Results}

We use an example of a range image to illustrate detailed results and to compare them to those obtained by other authors. Latter, we introduce the results of correct detection using the overall set of test images. The test image named abw.test.8 was considered, by several authors [56831] as a typical image in order to show visual results of segmentation, and to compare the involved methods. Obtained results with this image are presented in Fig. 2, Fig. 2a shows the rendered range image. Fig. 2b, 2 $\mathrm{c}$ 2 $\mathrm{d}$ and $2 \mathrm{k}$ show image segmentation of respectively USF, WSU, UB and UE methods. Fig. 2f presents the segmentation result obtained by our method.

Table 1. Comparison results with abw.test. 8 image for $T=80 \%$

\begin{tabular}{ccccccc}
\hline Method & $\begin{array}{c}\text { GT } \\
\text { region }\end{array}$ & $\begin{array}{c}\text { Correct } \\
\text { detection }\end{array}$ & $\begin{array}{c}\text { Over- } \\
\text { segmentation }\end{array}$ & $\begin{array}{c}\text { Under- } \\
\text { segmentation }\end{array}$ & Missed Noise \\
\hline USF & 21 & 17 & 0 & 0 & 4 & 3 \\
WSU & 21 & 12 & 1 & 1 & 6 & 4 \\
UB & 21 & 16 & 2 & 0 & 3 & 6 \\
UE & 21 & 18 & 1 & 0 & 2 & 2 \\
MABIS & 21 & 17 & 1 & 0 & 1 & 1 \\
\hline
\end{tabular}

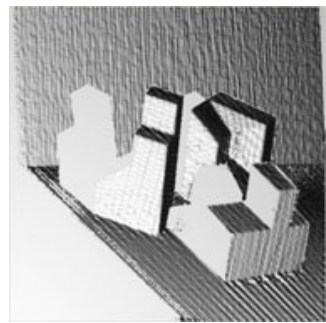

(a)

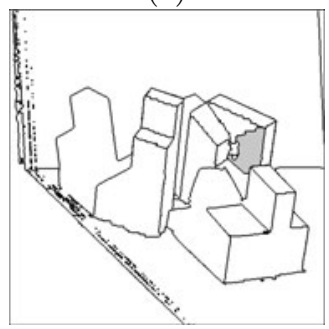

(d)

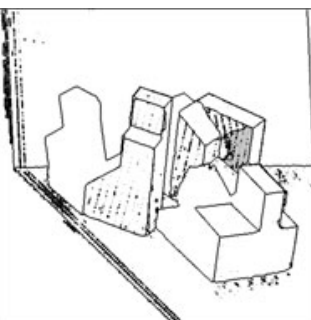

(b)

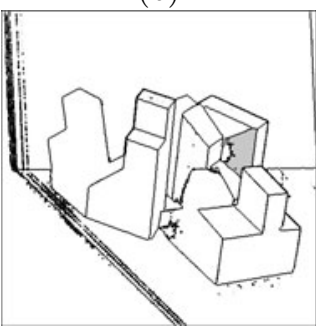

(e)

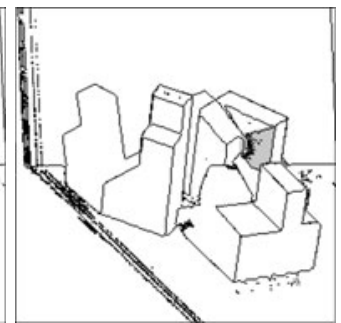

(c)

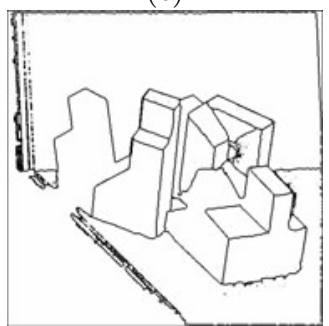

(f)

Fig. 2. Segmentation results of abw.test. 8 image. (a) Rendered Range image; (b) USF result; (c) WSU result; (d) UB result; (e) UE result; (f) MABIS result. 


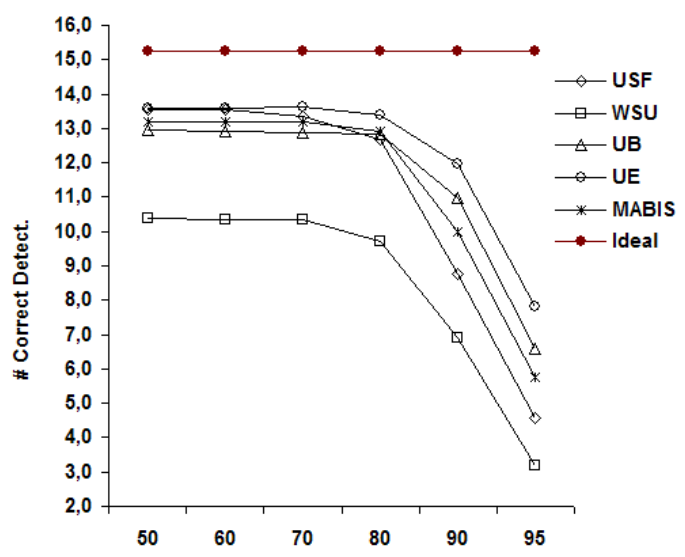

Fig. 3. Average results of correctly detected regions of all methods, according to the compare tool tolerance $T ; 0.5<T \leq 1.0$

We can see in Table 1 that the number of correct detected regions is equivalent to those obtained with the UE and USF algorithms, that scored better than UB and WSU. Our results for all incorrect detected regions are better than those corresponding to all the involved algorithms.

For the overall set of test images, Fig. 3 shows the average numbers of correctly detected regions, according to the compare tool tolerance $T ; T \in\{51 \%$, $60 \%, 70 \%, 80 \%, 90 \%, 95 \%$. Results show that the number of correctly detected regions by our method is in average equivalent to UE and USF algorithms, and better than those of UB and WSU ones. It scored higher than WSU for all the values of the compare tool tolerance $T$. It scored higher than USF for $T>70 \%$, and better than UB for $T<90 \%$.

\section{Conclusion}

In this paper we have presented an unsupervised and distributed approach for image segmentation, with application to range images. The distributed entities were modeled as autonomous and situated agents. Each agent performs region growing and image smoothing starting from a region seed, selected according to a quality criterion. Agents can share pixels, and thus must exchange information in order to better label pixels. Several tests and comparisons were performed using real images. Obtained experimental results has allowed to validate the proposed approach which provides an efficient region-based range image segmentation. In futur work, we plan to test the approach with other types of images, including range images containing curved objects, 2D grey-level, and color images. 


\section{References}

1. Bab Hadiashar, A., Gheissari, N.: Range image segmentation using surface selection criterion. IEEE Transactions on Image Processing 15(7), 2006-2018 (2006)

2. Ballet, P., Rodin, V., Tisseau, J.: Edge detection using a multiagent system. In: 10th Scandinavian Conference on Image Analysis, Lapeenranta, Finland, pp. 621626 (1997)

3. Ding, Y., Ping, X., Hu, M., Wang, D.: Range image segmentation based on randomized hough transform. Pattern Recognition Letters 26(13), 2033-2041 (2005)

4. Fan, T.J., Medioni, G.G., Nevatia, R.: Segmented description of 3-D surfaces. IEEE Journal of Robotics and Automation 3(6), 527-538 (1987)

5. Hoover, A., Jean-Baptiste, G., Jiang, X., Flynn, P.J., Bunke, H., Goldgof, D.B., Bowyer, K.W., Eggert, D.W., Fitzgibbon, A.W., Fisher, R.B.: An experimental comparison of range image segmentation algorithms. IEEE Transactions on Pattern Analysis and Machine Intelligence 18(7), 673-689 (1996)

6. Jiang, X., Bunke, H.: Edge detection in range images based on Scan Line approximation. Computer Vision and Image Understanding 73(2), 183-199 (1999)

7. Jones, J., Saeed, M.: Image enhancement, an emergent pattern formation approach via decentralised multi-agent systems. Multiagent and Grid Systems Journal (ISO Press) Special Issue on Nature inspired systems for parallel, asynchronous and decentralised environments 3(1), 105-140 (2007)

8. Li, S., Zhao, D.: Gradient-based polyhedral segmentation for range images. Pattern Recognition Letters 24(12), 2069-2077 (2003)

9. Liu, J., Tang, Y.Y.: Adaptive image segmentation with distributed behavior-based agents. IEEE Transactions on Pattern Analysis and Machine Intelligence 21(6), 544-551 (1999)

10. Richard, N., Dojat, M., Garbay, C.: Automated segmentation of human brain MR images using a multi-agent approach. Artificial Intelligence in Medicine 30(2), 153 176 (2004)

11. Rodin, V., Benzinou, A., Guillaud, A., Ballet, P., Harrouet, F., Tisseau, J., Le Bihan, J.: An immune oriented multi-agent system for biological image processing. Pattern Recognition 37(4), 631-645 (2004) 